\title{
Leptin contributes to the taxol chemoresistance in epithelial ovarian cancer
}

\author{
FEI GU ${ }^{1,2^{*}}$, HAO ZHANG ${ }^{3 *}$, LIANGQING YAO $^{3}$, SHUHENG JIANG $^{4}$, HUAN LU $^{1,2}$, \\ XIN XING ${ }^{2}$, CANCAN ZHANG ${ }^{1,2}$, PENGCHENG JIANG ${ }^{5}$ and RONG ZHANG ${ }^{1,2}$ \\ ${ }^{1}$ The Third School of Clinical Medicine, Southern Medical University, Guangzhou, Guangdong 510500; \\ ${ }^{2}$ Department of Obstetrics and Gynecology, Fengxian Hospital, Southern Medical University, Shanghai 201499; \\ ${ }^{3}$ Department of Obstetrics and Gynecology, Obstetrics and Gynecology Hospital, Fudan University, Shanghai 200011; \\ ${ }^{4}$ State Key Laboratory of Oncogenes and Related Genes, Shanghai Cancer Institute, Renji Hospital, \\ Shanghai Jiao Tong University School of Medicine, Shanghai 200240; ${ }^{5}$ Department of Obstetrics and Gynecology, \\ The Affiliated Changzhou No. 2 People's Hospital of Nanjing Medical University, Changzhou, Jiangsu 213000, P.R. China
}

Received April 4, 2018; Accepted April 2, 2019

DOI: $10.3892 / \mathrm{ol} .2019 .10381$

\begin{abstract}
Epithelial ovarian cancer (EOC) is a gynecological malignancy with high morbidity. Treating EOC remains a challenge, as the pathogenesis of this disease remains unclear and chemoresistance is a common occurrence. A number of previous studies have revealed that obesity is closely associated with cancer and leptin, as a link between cancer and obesity, has become a focus of research in recent years. In the present study, survival database analysis demonstrated that leptin expression was associated with poor prognoses in patients treated with platinum and paclitaxel/docetaxel. A cell activity assay demonstrated that leptin reduced the chemosensitivity of ovarian cancer cells to paclitaxel/docetaxel Furthermore, flow cytometry results revealed that treatment with exogenous leptin reduced the proportion of ovarian cancer cells in $\mathrm{G} 2 / \mathrm{M}$ phase, which was significantly elevated following paclitaxel/docetaxel chemotherapy. It was also verified that transcription factor CCAAT/Enhancer Binding Protein $\alpha$ can bind to the upstream promoter region of leptin and activate its transcription in ovarian cancer cells. Together, these results suggest that leptin serves an important role in chemoresistance and may serve as a novel therapeutic
\end{abstract}

Correspondence to: Dr Rong Zhang, Department of Obstetrics and Gynecology, Fengxian Hospital, Southern Medical University, 6600 NanFeng Road, Shanghai 201499, P.R. China

E-mail: rongzhang1965@163.com

Dr Pengcheng Jiang, Department of Obstetrics and Gynecology, The Affiliated Changzhou No. 2 People's Hospital of Nanjing Medical University, 29 Xinglong Lane, Changzhou, Jiangsu 213000, P.R. China E-mail: jpc1972@163.com

Key words: leptin, epithelial ovarian cancer, paclitaxel, chemoresistance, CCAAT/Enhancer Binding Protein $\alpha$ target for ovarian cancer in patients treated with platinum and paclitaxel chemotherapy.

\section{Introduction}

Ovarian cancer has the highest morbidity and mortality of all malignant diseases affecting the female reproductive system. Worldwide, $>140,200$ fatalities occur due to ovarian cancer each year (1). Conventional treatments for ovarian cancer are cytoreductive surgery and platinum-based chemotherapy; however, the overall survival rate of patients with ovarian cancer has not greatly improved over the past 40 years $(2,3)$. Although first-line chemotherapy regimens, including platinum combined with paclitaxel, are effective in certain patients for first time treatment, $75 \%$ of patients relapse following treatment (4). At present, patient response to chemotherapy is difficult to predict and so it is important to identify an effective predictive biomarker for chemotherapeutic resistance (5).

In previous years, leptin has become a focus of research due to its association with cancer and obesity (6,7). Leptin, also known as obesity hormone, is a hormone-like cytokine secreted by fat cells $(8,9)$. It serves a vital role in maintaining the dynamic balance between energy and weight by affecting appetite and energy consumption, which in turn directly modulates fat storage and metabolism (10). Furthermore, leptin promotes the proliferation of various cells and tissues and serves an important role in regulating energy metabolism, immune response, trauma repair and angiogenesis $(11,12)$. It has previously been reported that leptin is highly expressed in a number of tissues, including malignant esophageal tumors (13), pancreatic cancer (14) and rectal carcinoma (15), and is increased in the serum of women with various gynecological malignancies, including ovarian cancer (16). Leptin has been reported to be involved in regulating the diverse malignant phenotypes of ovarian cancer by participating in the hypothalamus pituitary gonadal axis and in multiple signaling pathways $(17,18)$; however, the mechanisms of chemoresistance in ovarian cancer remain to be elucidated. 
In this study, the Kaplan-Meier (KM) Plotter database was analyzed and it was identified that the overall survival rate of patients with epithelial ovarian cancer treated with platinum plus paclitaxel/docetaxel chemotherapy was increased in the low leptin expression group compared with the high leptin expression group. In addition, the effect of leptin on the chemosensitivity of ovarian cancer cells was demonstrated and the underlying mechanism was investigated. In conclusion the results of the present study suggest that leptin may be used as a selection index or prognostic biomarker for chemoresistance in patients with ovarian cancer.

\section{Materials and methods}

Drugs and protein. Chemotherapeutic drugs (Cisplatin, DDP; Paclitaxel, PTX; Docetaxel, TXT; Gemcitabine, GCB; Topotecan, TPT; all from AMQUAR) were dissolved in DMSO (Gibco; Thermo Fisher Scientific, Inc.) and stored at $-80^{\circ} \mathrm{C}$. Recombinant Human Leptin (Bio-Techne) was reconstituted at $1 \mathrm{mg} / \mathrm{ml}$ in sterile $20 \mathrm{mM}$ Tris- $\mathrm{HCl}(\mathrm{pH} \mathrm{8.0)}$ and stored at $-80^{\circ} \mathrm{C}$.

Cell culture. Human epithelial ovarian cancer cell lines HO8910PM, OVCAR8, ES-2, SKOV-3 and IGROV-1 were obtained from the Cell Bank of the Chinese Academy of Sciences and OV-MZ-15 was stored in the State Key Laboratory of Oncogenes and Related Genes, Shanghai Cancer Institute. Cells cultured in RPMI-1640 medium (Beijing Solarbio Science \& Technology Co., Ltd.) containing 10\% fetal bovine serum (FBS; Gibco; Thermo Fisher Scientific, Inc.) and 1\% penicillin-streptomycin (Gibco; Thermo Fisher Scientific, Inc.). All cells were incubated at $37^{\circ} \mathrm{C}$ in an atmosphere containing $5 \% \mathrm{CO}_{2}$.

Drug sensitivity test. Briefly, ovarian cancer cells were seeded at $1 \times 10^{4}$ cells $/ \mathrm{ml}$ in 96 -well plates and cultured for $12 \mathrm{~h}$ in RPMI-1640 medium. Cells were subsequently incubated with Cell Counting Kit-8 (CCK-8; Dojindo Molecular Technologies, Inc.) reagents for $1 \mathrm{~h}$ and the initial optical density (OD) value (day 0) was measured at $450 \mathrm{~nm}$ using the Infinite M1000 PRO (Tecan Group, Ltd.). Cells were then treated with increasing concentrations of the chemotherapeutic drugs DDP, PTX, TXT, GCB and TPT (AMQUAR) diluted in complete medium and were placed in the incubator for $48 \mathrm{~h}$ The OD value was measured again $48 \mathrm{~h}$ later (day 2). The OD values were used to draw an $\mathrm{IC}_{50}$ curve using the GraphPad Prism5 software (GraphPad Software, Inc.). In further experiments, differences in the $\mathrm{IC}_{50}$ curve were compared between the negative control group (pre-incubated with complete medium for $30 \mathrm{~min}$ ) and the test group (pre-incubated with $40 \mathrm{nM}$ exogenous leptin recombinant protein for $30 \mathrm{~min}$ ).

Flow cytometry. To assess the cell cycle, blank cells (termed WT) were seeded in 6-well plates until they reached $70 \%$ confluence and were divided into six groups; three negative control groups (NC-1, NC-2 and $\mathrm{NC}-3$ ) and three test groups (TG-1, TG-2 and TG-3). After $12 \mathrm{~h}$, cells in the NC and TG groups were treated as follows. First, $200 \mu \mathrm{l}$ RPMI 1640 containing $10 \%$ FBS was added to two groups of cancer cells to serve as control groups and $200 \mu \mathrm{l} 40 \mathrm{nM}$ exogenous recombinant leptin protein to two other groups to give the test groups. After pre-incubation for $30 \mathrm{~min}, 1 \mathrm{ml}$ training solution (RPMI-1640 medium containing 10\% FBS) was added to the NC-1 (WT) group, $1 \mathrm{ml}$ PTX was added to the NC-2 $(\mathrm{WT}+\mathrm{PTX})$ group and $1 \mathrm{ml}$ TXT was added to the NC-3 $(\mathrm{WT}+\mathrm{TXT})$ group. Similarly, $1 \mathrm{ml}$ of culture without leptin was added to the TG-1 (WT + leptin) group, $1 \mathrm{ml}$ PTX was added to the TG-2 (WT + leptin + PTX) group and $1 \mathrm{ml} \mathrm{TXT}$ was added to the TG-3 (WT + leptin + TXT) group for 8 or 16 h. Following treatment, HO8910PM and OV-MZ-15 cells were harvested, fixed in ethanol for $2 \mathrm{~min}$ at room temperature, washed twice with PBS and stained with PI solution containing PI and RNAse for $30 \mathrm{~min}$ at room temperature in the dark, Cells were subsequently counted using a flow cytometer (BD FACSCalibur2; BD Biosciences) and analyzed using FlowJo_V10 software (Becton, Dickinson and Company).

Chromatin immunoprecipitation $(\mathrm{CH}-\mathrm{IP})$. The transcription factor CCAAT/enhancer-binding protein alpha (CEBPA) was predicted by ALGGEN-PROMO (http://alggen.lsi.upc.es/) and JASPAR (http://jaspar.genereg.net) websites. The 3,000 bp nucleic acid sequence of the leptin upstream promoter region was obtained from the UCSC database (http:/genome.ucsc. edu/) and 10 pairs of primers were designed (Sangon Biotech Co., Ltd.) for the $\mathrm{CH}$-IP experiment. WT cells were fixed with $4 \%$ formaldehyde for $10 \mathrm{~min}$ at room temperature and chromosomes were extracted using a CH-IP Assay kit (Thermo Fisher Scientific, Inc.). In the extracted liquid, immunoglobulin $\mathrm{G}$ (Abcam) was added to the NC groups, CEBPA-antibody (Abcam) was added to the TGs, and RNA polymerase II antibody (Thermo Fisher Scientific, Inc.) was added as the positive control group. Purified and enriched DNA- fragments were obtained following precipitation and cleaned of immune complexes. Ultimately, the DNA fragments of above cells were analyzed by polymerase chain reaction (PCR). The procedure used for PCR was as follows: Pre-denaturation at $95^{\circ} \mathrm{C}$ for $5 \mathrm{~min}$, denaturation at $95^{\circ} \mathrm{C}$ for $30 \mathrm{sec}$, annealing at $60^{\circ} \mathrm{C}$ for $30 \mathrm{sec}$, extension at $72^{\circ} \mathrm{C}$ for 35 cycles, and extension at $72^{\circ} \mathrm{C}$ for $10 \mathrm{~min}$.

Luciferase reporter assay. A total of 4 luciferase plasmids (Promega Corporation), leptin primordial plasmid-1/2, leptin mutant plasmid-1/2 and a CEBPA overexpression plasmid without luciferase were constructed. At the beginning of the experiment, CEBPA-overexpressing plasmids were transfected into target cells using leptin primordial/mutant plasmids and a Roche X-tremeGENE HP DNA Transfection Reagent kit (Roche Diagnostics GmbH). At 48 h later, cells were stained using the Dual-Glo Luciferase Assay System (cat. no. E2920; Promega Corporation) and the fluorescence intensity was measured using SpectraMax M5 (Molecular Devices LLC). Firefly luciferase activity was normalized to Renilla luciferase activity.

Gene set enrichment analysis (GSEA). A total of 69 cases of EOC patients treated with PTX were selected from the U133A Chip dataset from the TCGA database and were divided into the high expression (23 cases) and low expression groups (23 cases) according to leptin expression. GSEA was performed using gsea-3.0, downloaded from the GSEA 
Table I. The effect of leptin in epithelial ovarian cancer overall survival rate under different chemotherapy regimens.

\begin{tabular}{lcccc}
\hline Drugs & $\mathrm{N}$ & High expression of leptin & Low expression of leptin & Log rank P \\
\hline Paclitaxel & 220 & 108 & 112 & 0.0089 \\
Docetaxel & 108 & 52 & 56 & 0.0021 \\
Topotecan & 119 & 56 & 63 & 0.98 \\
Gemcitabine & 135 & 66 & 69 & 0.082 \\
Platin & 1,409 & 711 & 698 & 0.58 \\
\hline
\end{tabular}
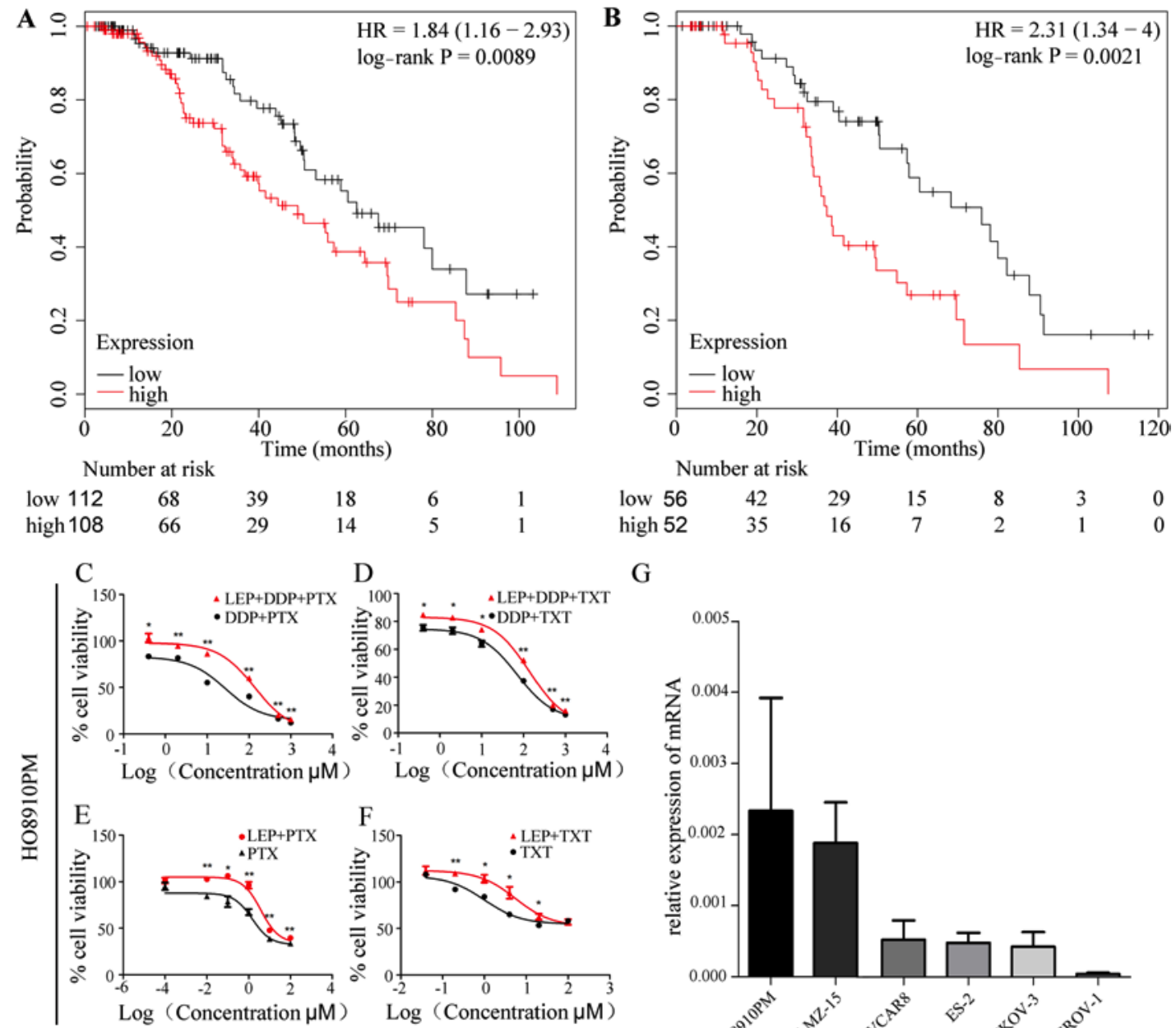

$\begin{array}{lllllll}\text { low } 56 & 42 & 29 & 15 & 8 & 3 & 0 \\ \text { high } 52 & 35 & 16 & 7 & 2 & 1 & 0\end{array}$

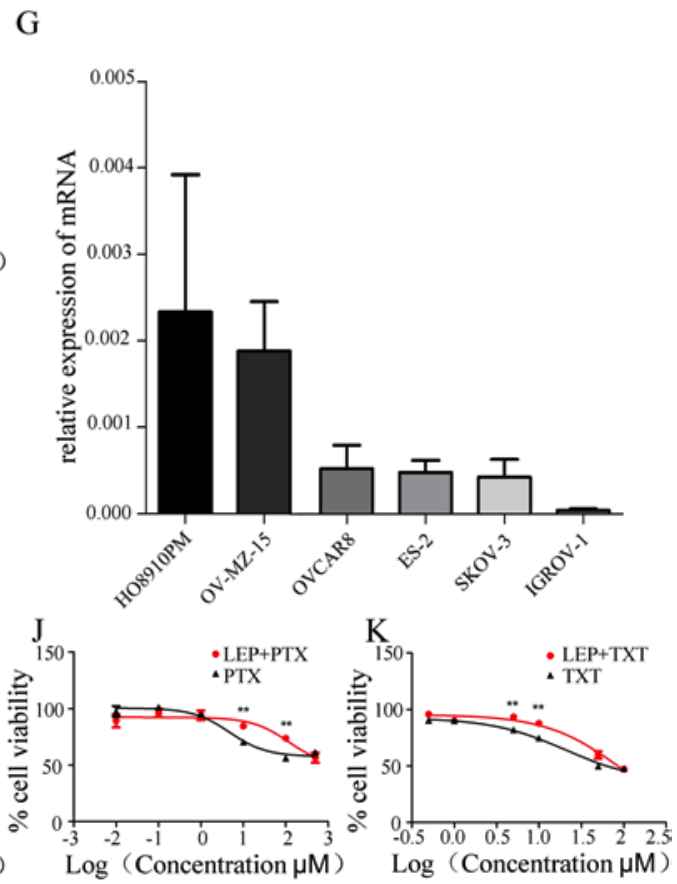

Figure 1. Leptin reduces the chemosensitivity of ovarian cancer cells to PTX/TXT. The prognosis of patients with epithelial ovarian cancer receiving (A) PTX $(\mathrm{P}=0.008)$ or $(\mathrm{B})$ TXT $(\mathrm{P}=0.0021)$ chemotherapy was analyzed based on leptin RNA expression level. CCK- 8 assay was performed to assess the cell viability of HO8910PM cells treated with DDP combined with (C) PTX/ (D) TXT with or without leptin. CCK-8 assay was performed to assess the HO8910PM cell survival percentage treated with (E) PTX/ (F) TXT with or without leptin. (G) The expression of leptin was highest in the HO8910PM and OV-MZ-15 cell lines. CCK-8 assay was performed to assess the cell viability of OV-MZ-15 cells treated with DDP combined with (H) PTX/ (I) TXT with or without leptin. CCK-8 assay was performed to assess the cell viability of OV-MZ-15 cells treated with (J) PTX/ (K) TXT with or without leptin. Data are presented as the means \pm standard deviation. ${ }^{*} \mathrm{P}<0.05,{ }^{* *} \mathrm{P}<0.01$ and ${ }^{* * *} \mathrm{P}<0.001$.

database (http://software.broadinstitute.org/gsea/index.jsp )with the built-in standard datasets.
Other sources of data. The database NCBI (https://www.ncbi. nlm.nih.gov/pubmed/), GENECARDS (http://www.genecards. 
Table II. The $\mathrm{IC}_{50}$ values and corresponding concentrations of commonly used chemotherapeutic agents in ovarian cancer cells.

\begin{tabular}{lccccccc}
\hline & \multicolumn{2}{c}{ HO8910PM } & & \multicolumn{2}{c}{ OV-MZ-15 } \\
\cline { 2 - 3 } Drugs & Concentration gradient & $\mathrm{IC}_{50}$ & Unit & & Concentration gradient & $\mathrm{IC}_{50}$ & Unit \\
\hline Paclitaxel & $0.001,0.01,0.1,1,10,100$ & 6.963 & $\mathrm{nM}$ & & $0.01,0.1,1,10,100,500$ & 2.315 & $\mathrm{nM}$ \\
Docetaxel & $0.04,0.2,1,4,20,100$ & 2.569 & $\mathrm{nM}$ & & $0.5,1,5,10,50,100$ & 3.001 & $\mathrm{nM}$ \\
Cisplatin & $0.4,2,10,100,500,1000$ & 82.66 & $\mathrm{uM}$ & & $1,10,100,1000,2000,5000$ & 23.02 & $\mathrm{uM}$ \\
Gemcitabine & $1,2,4,10,20,40$ & 12.882 & $\mathrm{nM}$ & & $20,50,100,500,1000,2000$ & 151.2 & $\mathrm{nM}$ \\
Topotecan & $0.01,0.1,5,100,1000,2000$ & 20.62 & $\mathrm{uM}$ & & $10,20,200,800,3200,6400$ & 174.0 & $\mathrm{nM}$ \\
\hline
\end{tabular}

org), CBioPortal (http://www.cbioportal.org) and Oncomine Main (https://www.oncomine.org/resource/main.html) were used for basic information acquisition. The Kaplan-Meier estimator (http://kmplot.com/analysis/index.php?p=service \&cancer=ovar) was used to estimate survival. The website ALGGEN-PROMO (http://alggen.lsi.upc.es) and JASPAR (http://jaspar.genereg.net) were used to predict transcriptional factors. The database Gene Set Enrichment Analysis (GSEA; http://software.broadinstitute.org/gsea/ind ex.jsp) was used to analysis the enrichment gene set.

Statistical analysis. All experiments were repeated three times. Data are presented as the means \pm standard deviation. Statistical analysis was performed using GraphPad PRISM (version 5.0; GraphPad Software, Inc.) Differences between groups were assessed using analysis of variance with the Dunnett's least significant difference post-hoc tests. $\alpha=0.05$ and $\mathrm{P}<0.05$ was considered to indicate a statistically significant difference.

\section{Results}

Leptin is associated with a poor prognosis in patients treated with platinum combined with PTX/TXT. Using the Kaplan-Meier method, the overall survival rate of 1,656 ovarian cancer patients treated using different chemotherapy regimens was analyzed, with the median leptin expression as the boundary. The results indicated that the overall survival rate was significantly decreased in the leptin high expression group $(n=108)$ compared with the low expression group $(n=112)$ in. $(n=220 ; P=0.0089$; Fig. 1A and Table I). Similar results were observed in patients treated with platinum added TXT (n=108; P=0.0021; Fig. 1B, Table I).

No significant differences in overall survival rate were observed between the high expression and low expression groups in patients who received platinum in chemotherapy combined with TPT or GCB $(\mathrm{P}=0.98 ; \mathrm{P}=0.082$; Table I).

Leptin increases the chemoresistance of ovarian cancer cells to PTX/TXT. Primarily, the expression level of leptin was compared between six ovarian cancer cell lines (HO8910PM, OV-MZ-15, OVCAR8, ES-2, SKOV-3 and IGROV-1) using RT-qPCR. The results demonstrated that the expression of leptin was highest in the two cell lines HO8910PM and OV-MZ-15. In this study, to better simulate the situation in vivo, these two strains were selected for in vitro assays (Fig. 1G). CCK-8 was used to analyze cell viability following treatment with five different chemotherapeutic drugs and to determine the determined $\mathrm{IC}_{50}$ values (Table II).

Cells were next treated with exogenous leptin for $30 \mathrm{~min}$ followed by DDP+PTX/TXT for $48 \mathrm{~h}$. The data demonstrated that the survival rate of the two cell lines was significantly increased in the TG compared with the $\mathrm{NC}$ group $(\mathrm{P}<0.05$; Fig. 1C, D, H and I). The results demonstrated that the addition of leptin reduced the chemosensitivity of ovarian cancer cells to DDP and PTX/TXT treatment, which is in agreement with the database analysis.

In order to further investigate which drug is directly influenced by leptin in combination chemotherapy, the cells were cultured for $48 \mathrm{~h}$ with DDP (TG1) or PTX/TXT alone (TG2 and TG-3) under the same condition used previously. The results revealed that there were no significant differences in cell activity in the TG1 groups (leptin + DDP) compared with $\mathrm{NC}-1$ ( $\mathrm{P}>0.05$, data not shown). On the contrary cell activity was significantly increased in TG2 (leptin + PTX) and TG3 (leptin + TXT) compared with NC-2 and NC-3, respectively $(\mathrm{P}<0.05$; Fig. 1E, F, J and $\mathrm{K})$. These results suggest that high leptin expression may contribute to the chemoresistance of ovarian cancer cells treated with PTX/TXT.

Leptin reverses the inhibitory effect of PTX/TXT on the G2/M phase of ovarian cancer cells. To further investigate the effect of leptin on the reduction of PTX/TXT sensitivity in ovarian cancer cells, changes in the cell cycle were measured using a flow cytometer under several conditions. Compared with $\mathrm{NC}-1$, the proportion of cells in the $\mathrm{G} 2 / \mathrm{M}$ phase was increased significantly in the NC-2 group at 3,6 and $18 \mathrm{~h}(\mathrm{P}<0.05$, $\Delta \mathrm{n}>10 \%$; Fig. 2). PTX and TXT were also demonstrated to block cell division of ovarian cancer cells in G2/M phase. No significant differences in cell cycle distribution were observed in the NC-1 and TG-1 groups, suggesting that leptin did not directly affect the cell cycle. As expected, the proportion of cells in G2/M phase was decreased in TG-2 cells compared with the NC-2 group. Similar results were also observed when comparing TG-3 and NC-3. In the present study, leptin clearly reversed the inhibitory effects of PTX/TXT on the G2/M phase proportion of ovarian cancer cells, which suggested that it may reduce the sensitivity of ovarian cancer cells to PDX/TXT treatment.

CEBPA binds the upstream promoter region of leptin to initiate leptin transcription in ovarian cancer cells. To investigate the potential molecular mechanism of leptin in the development 
A

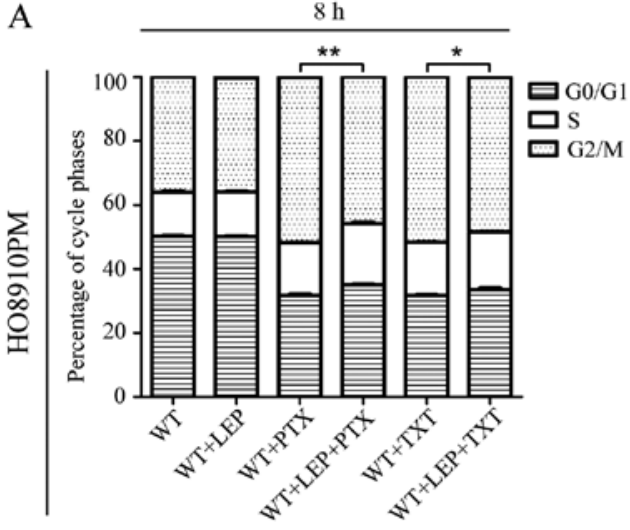

$\mathrm{C}$

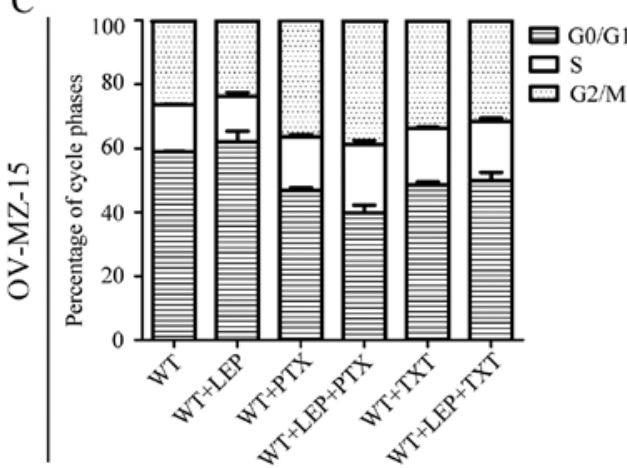

B

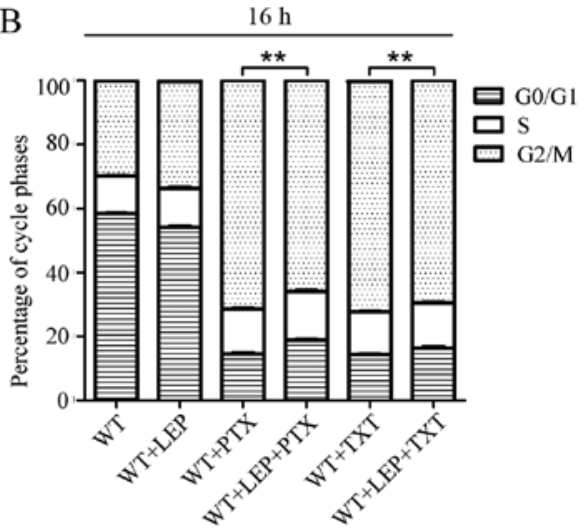

D

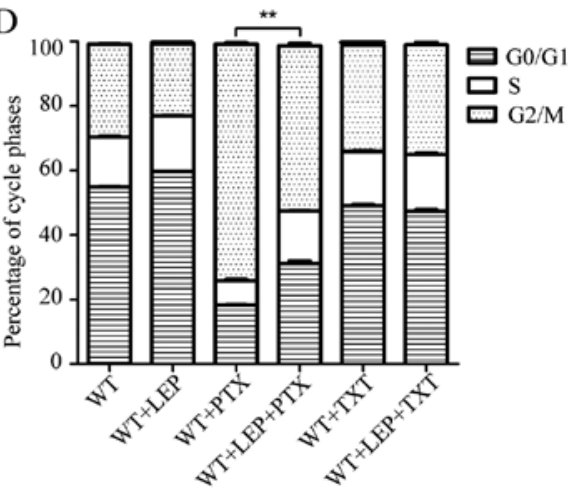

Figure 2. Effects of leptin on the cell cycle distribution of ovarian cancer cells following PTX/TXT treatment. Flow cytometry at (A) 8 and (B) $16 \mathrm{~h}$ was used to assess the effect of PTX on the cell cycle distribution of ovarian cancer cells in the presence of leptin. Flow cytometry at (C) 8 and (D) $16 \mathrm{~h}$ was used to assess the effect of TXT on the cell cycle distribution of ovarian cancer cells in the presence of leptin. ${ }^{*} \mathrm{P}<0.05,{ }^{* * *} \mathrm{P}<0.01$.

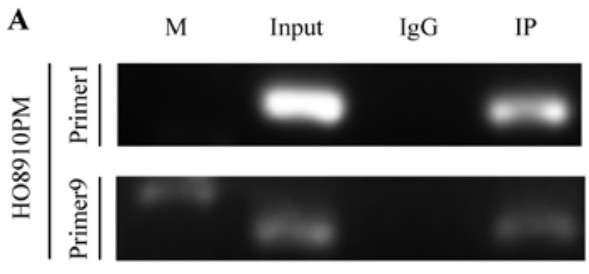

B Leptin promoter lucifrase MUT1
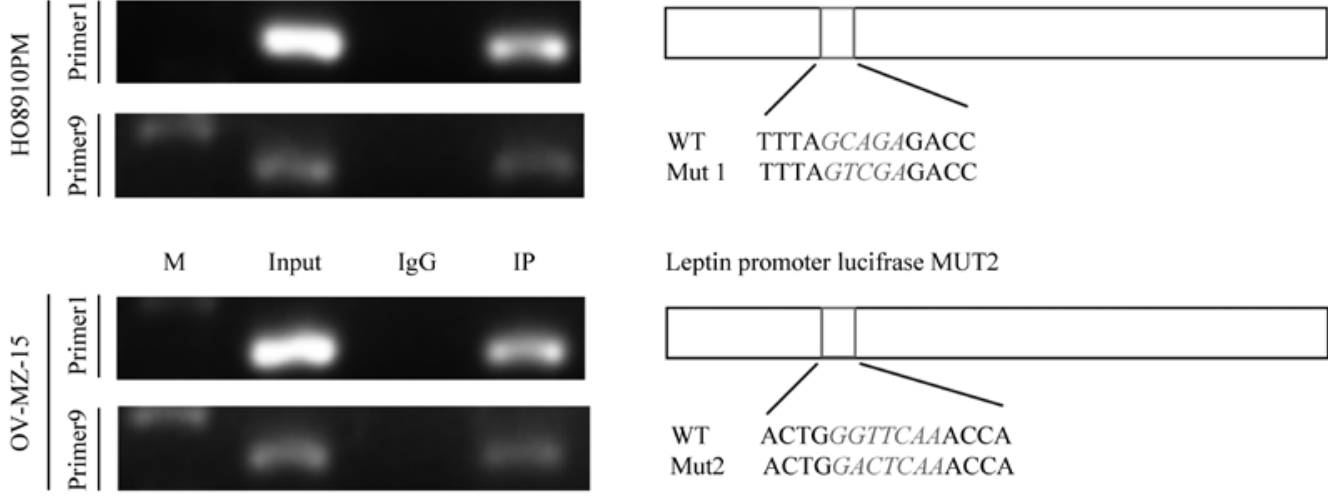

Leptin promoter lucifrase MUT2

C

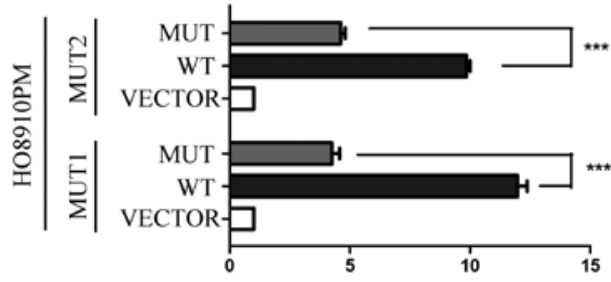

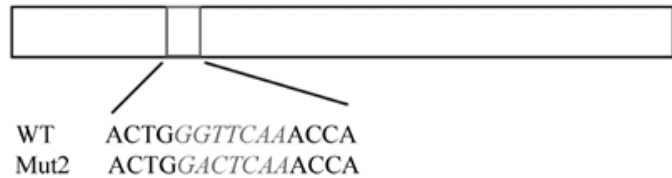

D

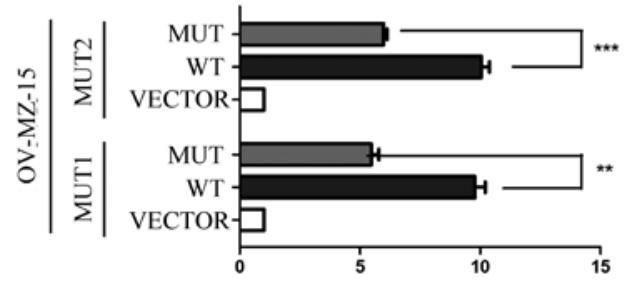

Figure 3. CEBPA initiates the transcription of leptin in ovarian cancer cells. (A) A chromatin immunoprecipitation-polymerase chain reaction assay was performed for HO8910PM and OV-MZ-15 cells. Total DNA (Input), IgG and CEBPA immunoprecipitated DNA were amplified using a series of primers covering the 3,000 bp sequence upstream of the leptin transcription start site. Primer 1 and 9 flanking sequences can be immunoprecipitated with CEBPA. (B) Site directed mutagenesis of the upstream loci of leptin. Leptin vector, leptin-WT or mutant plasmids leptin-MUT1 and 2 were transfected into CEBPA overexpression or control (C) HO8910PM and (D) OV-MZ-15 cells. The pRLTK Renilla luciferase expression plasmid was co-transfected as the internal control. Luciferase activities were normalized to Renilla activities. MUT, mutant; WT, wild-type; CEBPA, CCAAT/enhancer-binding protein $\alpha$; IgG, immunoglobulin $\mathrm{G} .{ }^{* *} \mathrm{P}<0.01,{ }^{* * *} \mathrm{P}<0.001$. 
of epithelial ovarian cancer, potential leptin transcription factors were first investigated using the ALGGEN-PROMO and JASPAR websites. The results identified a number of possible transcription factors, including Jun Proto-Oncogene, Activating Transcription Factor 2, CCAAT/Enhancer Binding Protein $\beta$, Zinc Finger E-Box Binding Homeobox 1 and CEBPA. CEBPA, a transcription factor related to fat production, attracted the attention of the authors of the present study. To verify the database results, a 0-3,000 bp nucleic acid sequence was searched for in the leptin upstream promoter region using the gene promoter analysis database and 10 pairs of primers were designed for a CH-IP assay (Table III). The $\mathrm{CH}$-IP results demonstrated that there are at least two sites in the leptin promoter region to which CEBPA can bind (primer 1 and primer 9, the range from $301 \mathrm{bp}$ to $655 \mathrm{bp}$ and from 2,742 bp to 3,000 bp on ORF) (Fig. 3A).

In the next experiment, $\mathrm{CH}$-IP results were confirmed using a luciferase reporter gene assay. Luciferase activity was detected with or without a fixed-point mutation of leptin following transfecting the cells with a CEBPA-overexpressing plasmid (Mut1/Mut2; Fig. 3B). Activity values were distinctly decreased in the mutant group compared with the wild-type group (HO8910PM-MUT1, P=0.000114696; HO8910PM-MUT2, $\mathrm{P}=2.646 \mathrm{E}-05 ; \mathrm{OV}-\mathrm{MZ}-15-\mathrm{MUT} 1, \quad \mathrm{P}=0.001271496$; OV-MZ-15-MUT2, $\mathrm{P}=0.000403456$; Fig. 3C and D). This phenomenon suggests that CEBPA-overexpression specifically increased the expression of leptin at the DNA level. Therefore it was hypothesized that the transcriptional factor CEBPA positively regulates the expression of leptin.

High leptin expression leads to significant enrichment of multiple hallmarks in cancer gene sets. To determine how leptin enhances the chemotherapeutic resistance of epithelial ovarian cancer to PTX/TXT, 309 cases from the TCGA database of patients with epithelial ovarian cancer and complete clinical data were sorted by leptin expression, and in-depth analyses of 69 patients undergoing platinum-combined PTX chemotherapy regimens was conducted. These patients were arranged by leptin expression, from high to low: The $33 \%$ of cases with the lowest expression were termed the low expression group and the highest $33 \%$ of cases were termed the high expression group ( $\mathrm{P}<0.001$; Fig. 4A). A heatmap revealed that when leptin expression is increased, 20 genes are downregulated and 35 are upregulated (Fig. 4B). The following GSEA analysis demonstrated a clear enrichment in tumor marker genes and KEGG gene set in the high expression group (Fig. 4C; Table IV), including the hallmark Epithelial-to-Mesenchymal transition (EMT; Fig. 4D), TNF $\alpha$-signaling-, via NFאB (Fig. 4E), Kras-signaling-up (Fig. 4F), HALLMARK_HYPOXIA (Fig. 4G), KEGG-ECM-RECEPTOR-INTERACTION (Fig. 4H) and HALLMARK- IL6-JAK-STAT3-SIGNALING (Fig. 4I). Among these, the highest scoring gene set was EMT, which serves an important role in phenotypic change during normal cell canceration and leads to chemotherapy resistance in cancer cells.

\section{Discussion}

Drug resistance has been persistent in the treatment of human diseases for a number of years and is primarily responsible for
Table III. Additional: Primer sequences in for chromatin immunoprecipitation.

\begin{tabular}{ll}
\hline Primers & \multicolumn{1}{c}{ Sequences } \\
\hline Primer 1 & \\
Forward & CTATTTGCTGCCTTGAATTATTCCTCCTC \\
& TCC \\
Reverse & ATCAACTGCAGGGCAGGGA
\end{tabular}

Primer 2

Forward TCCATCAACCTCAGGAACCGAGCTCC

Reverse TTCCCAATATCTTGTCTTCCGTTCTTCCC CAGTC

Primer 3

Forward GACTTGGAGTTTTCTATGACTGGGGAAG AACGGA

Reverse ACAAGCCCCCTCTATCACCACTGATAAT ATGCTTCAG

Primer 4

Forward ATTTCCAGCATCCACTGAAGCATATTATC AGTG

Reverse CTCTATTAGCTACTTGTTACCTGAATAAT ACACCAAATGTTTGTGC

Primer 5

Forward CTGTTGCACAAACATTTGGTGTATTATTC AGGTAACAAG

Reverse GAATAGGAGTCAACTTGCCCAAAGTCA AACAG

Primer 6

Forward TCCTGACTCTGTCATGGACCTGTTTG

Reverse CAATGATCCATGCTAAGTATATGTGCATG AGCC

Primer 7

Forward

TGCCATCTCCAGAACCGTCA

Reverse GGGCTCCCTGGAAGAAGTGT

Primer 8

Forward TGTTATGCTCTCTCCCGCCA

Reverse GGGCCTTTACCACTTGCTTCC

Primer 9

Forward

TGCTAGTGGGATTCAGGCTCC

Reverse CTGTGAGGCCAGGGTGTGA

Primer 10

Forward

TGTCCATTTGATCACACCCTGGC

Reverse

antineoplastic drugs failing to kill tumor cells during cancer treatment (19). Ovarian cancer has one of the highest mortality rates among malignancies due to its typically late diagnosis, high recurrence rate and chemotherapeutic resistance (20). Therefore, chemosensitivity and drug resistance analyses are essential for selecting appropriate chemotherapeutic regimens and in survival assessments for patients with epithelial ovarian cancer (21).

There are multiple reasons why chemotherapeutic resistance in ovarian cancer is not yet well understood, as well 

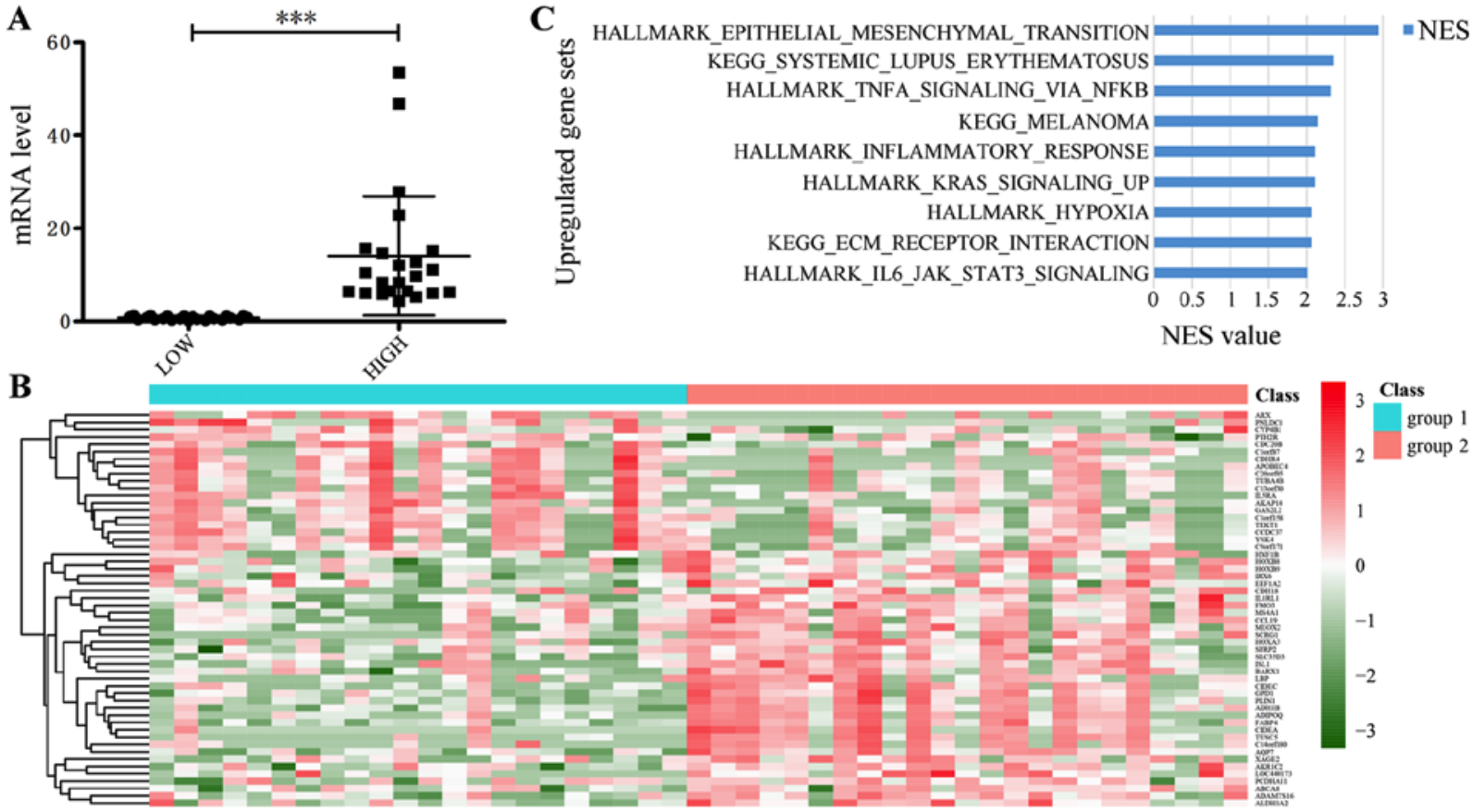

D

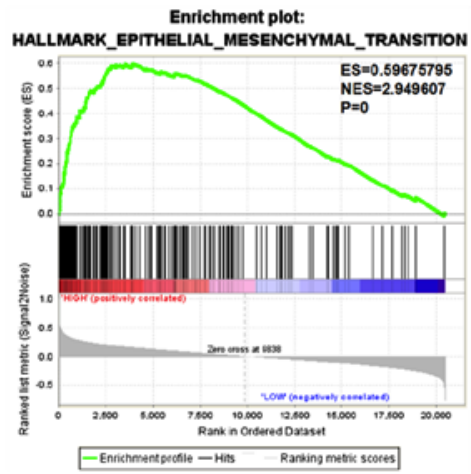

G

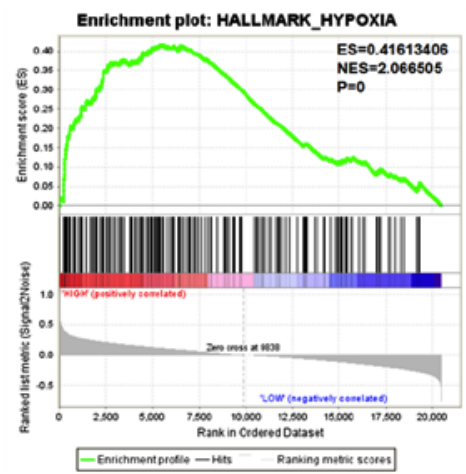

E

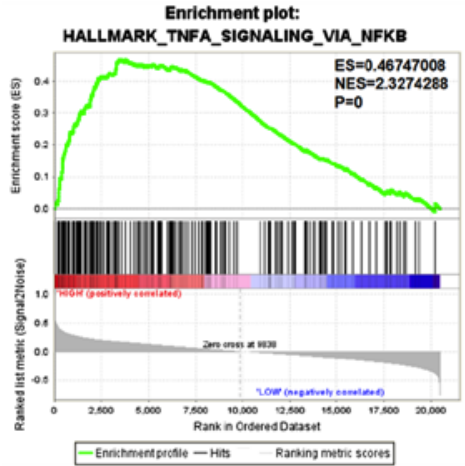

H Enrichment plot: KEGG_ECM_RECEPTOR_INTERACTION

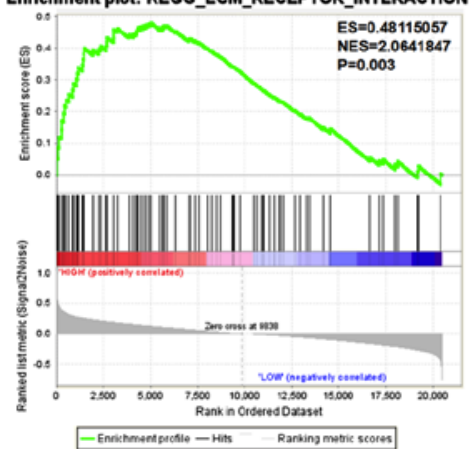

F Enrichment plot: HALLMARK_KRAS_SIGNALING_UP

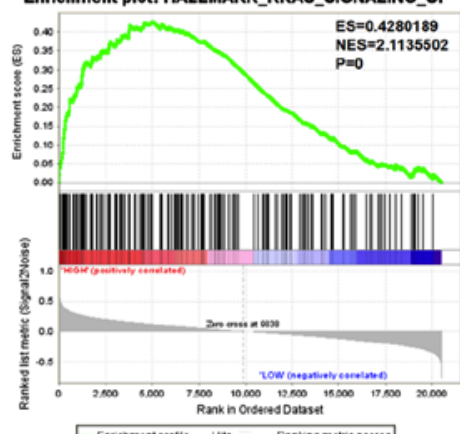

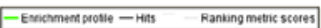

I

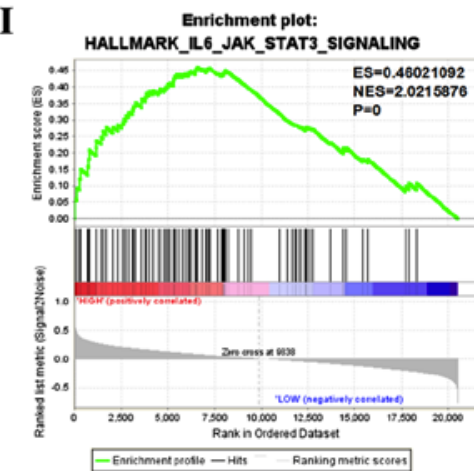

Figure 4. High leptin expression is correlated with a significant enrichment of multiple hallmark of cancer gene sets. (A) A total of 46 patients with ovarian cancer treated with PTX were assessed and divided into two groups according to leptin expression. ${ }^{* * *} \mathrm{P}<0.001$. (B) A heatmap of differentially expressed genes between groups associated with low leptin expression one and two was constructed group 1, leptin low expression; group 2, leptin high expression; 'red' means upregulation and 'green' means downregulation). (C) Results from the gene set enrichment analysis revealed the leptin-associated enrichment gene sets with the highest NES value. (D-I) The test leptin-associated enrichment gene sets were closely associated with tumor progression and chemoresistance and included the following hallmark: (D) HALLMARK_EPITHELIAL_MESENCHYMAL_TRANSITION; (E) HALLMARK_TNFA_SIGNALING_VIA_NFKB; (F) HALLMARK_KRAS_SIGNALING_UP; (G) HALLMARK_HYPOXIA; (H) KEGG_ECM_RECEPTOR_INTERACTION; and (I) HALLMARK_ IL6_JAK_STAT3_SIGNALING NES, Normalized Enrichment Score.

as chemoresistance: i) The existence of multidrug resistance efflux pumps that accelerate drug metabolism (22); ii) changes in cell surface receptors/carriers that result in decreased drug influx (23); iii) drug-mediated DNA repair that enhances the antagonism of most chemotherapeutic drugs (24); and iv) lipid metabolism-related genes that are involved in the EMT of cancer cells and mediate a reduction in drug sensitivity (25).

Cuello et al (26) analyzed 83 consensus driver genes and 143 lipid metabolism-associated genes in 681 patients 
Table IV. Enrichment gene sets in the high leptin expression group.

\begin{tabular}{lccc}
\hline Gene set & NES & q-value & P-value \\
\hline HALLMARK_EPITHELIAL_MESENCHYMAL_TRANSITION & 2.950 & 0 & 0 \\
KEGG_SYSTEMIC_LUPUS_ERYTHEMATOSUS & 2.355 & 0 & 0 \\
HALLMARK_TNFA_SIGNALING_VIA_NFKB & 2.327 & 0 & 0 \\
KEGG_MELANOMA & 2.158 & 0 & 0 \\
HALLMARK_INFLAMMATORY_RESPONSE & 2.118 & 0 & 0 \\
HALLMARK_KRAS_SIGNALING_UP & 2.114 & 0 & 0 \\
HALLMARK_HYPOXIA & 2.067 & 0 & 0.003 \\
KEGG_ECM_RECEPTOR_INTERACTION & 2.064 & 0.002 & 0 \\
HALLMARK_IL6_JAK_STAT3_SIGNALING & 2.022 & 0 & 0 \\
HALLMARK_ALLOGRAFT_REJECTION & 1.998 & 0 & 0.001 \\
HALLMARK_IL2_STAT5_SIGNALING & 1.921 & 0.000 & 0.031 \\
KEGG_CYTOKINE_CYTOKINE_RECEPTOR_INTERACTION & 1.913 & 0.013 & 0.004 \\
HALLMARK_PANCREAS_ß_CELLS & 1.904 & 0.000 & 0.042 \\
KEGG_REGULATION_OF_ACTIN_CYTOSKELETON & 1.896 & 0.014 & 0.005 \\
HALLMARK_APICAL_JUNCTION & 1.896 & 0.001 & 0.006 \\
HALLMARK_ANGIOGENESIS & 1.858 & 0.001 & 0.007 \\
HALLMARK_CHOLESTEROL_HOMEOSTASIS & 1.828 & 0.001 & 0.007 \\
HALLMARK_MYOGENESIS & 1.823 & 0.001 & 0.012 \\
HALLMARK_ADIPOGENESIS & 1.754 & 0.001 & 0.012 \\
HALLMARK_GLYCOLYSIS & 1.753 & 0.001 & 0.012 \\
HALLMARK_MTORC1_SIGNALING & 1.751 & 0.001 & 0.015 \\
HALLMARK_TGF_B_SIGNALING & 1.742 & 0.002 & 0.022 \\
HALLMARK_UV_RESPONSE_DN & 1.716 & 0.002 & 0.038 \\
HALLMARK_P53_PATHWAY & 1.664 & 0.004 & 0.047 \\
HALLMARK_XENOBIOTIC_METABOLISM & 1.628 & 0.004 & 0.047 \\
HALLMARK_APOPTOSIS & & 0.004 & \\
\hline
\end{tabular}

with high-grade serous ovarian cancer, obtained from the ICGC database, using gene chip and reverse protein chip clustering analyses. This study demonstrated that certain genes associated with obesity and lipid metabolism disorder may have significant effects on the prognosis of patients with ovarian cancer. It was also reported that at the same clinical stage of ovarian cancer, compared with non-obese patients, the clinical prognosis of obese patients is considerably poorer.

Leptin is a secretory protein that is synthesized mainly in white adipocytes. In addition to controlling the energy metabolism and weight balance of an organism, leptin has multiple endocrine functions and participates not only in the regulation of immune and inflammatory responses, hematopoiesis, angiogenesis, reproduction, bone formation and wound healing, but also in cancer development through regulating diverse malignant phenotypes in cancer cells (10-16). Previous studies have reported that the high expression of leptin and its receptor impacts the proliferation, migration and invasion of ovarian cancer cells by activating the JAK/STAT, MAPK, PI3K/AKT and RhoA/ROCK signaling pathways $(27,28)$.

Based on the KM Plotter database, it was confirmed that the overall survival rate of patients with high leptin expression was decreased compared with patients with low leptin expression, following treatment with platinum plus PTX/TXT. These results are consistent with previous reports by Cuello et al (26). However, similar results were not recorded for cases of DDP combined with GCB or TPT, for which the present study hypothesized that other mechanisms underlie the activity of chemotherapeutics.

DDP is known to directly damage DNA structure and affect its function. GCB is a cell cycle-specific drug that acts primarily in the $S$ phase and prevents cells entering the $S$ phase from G1. Additionally, TPT can hinder the re-linking of single strands of broken DNA and cause damage to double-stranded DNA. However, the mechanism by which PTX/TXT kills tumor cells relies on block cell division in the $\mathrm{G} 2 / \mathrm{M}$ phase through the inhibition of microtubule depolymerization. CCK-8 assays demonstrated that the addition of an exogenous recombinant leptin protein decreased the cytotoxicity of PTX/TXT towards ovarian cancer cells. Furthermore, flow cytometry demonstrated that the addition of leptin to cells treated with PTX/TXT significantly reduced the proportion in the G2/M phase. Therefore, it was hypothesized that high levels of leptin may reduce the sensitivity of ovarian cancer cells to PTX/TXT treatment via blocking the effect exerted by PTX/TXT on microtubules. Leptin expression levels may be a good predictor of chemoresistance when guiding treatment and/or evaluating prognosis in patients 
with ovarian cancer receiving platinum plus PTX/TXT chemotherapy.

The mechanism of chemotherapeutic resistance in ovarian cancer is very complex. To investigate potential mechanisms by which high leptin expression reduces ovarian cancer cell sensitivity to PTX/TXT treatment, 69 cases were identified in which patients with ovarian cancer underwent PTX chemotherapy and analyzed leptin mRNA expression data using GSEA analysis. It was demonstrated that 26 gene sets were significantly enriched in the high leptin expression group. Among the enriched sets, the highest scoring was the EMT gene set. EMT is a biological process in which polarized epithelial cells transform into mesenchymal cells. This process is not only common in cancer initiating cells, which enhances their invasive and migratory abilities, but is also closely associated multiple drug resistance in human tumors (29-31). Therefore, it was hypothesized that high leptin expression may also lead to PTX resistance through the activation of EMT in ovarian cancer cells. The authors are interested in investigating their correlations and the underlying mechanisms in their future work.

In addition, using the ALGGEN and JASPAR websites, it was predicted that CEBPA may be a transcription factor for leptin. CHIP and luciferase reporter assays confirmed that CEBPA can bind the upstream promoter region of leptin to initiate gene transcription. CEBPA is a CCAAT protein binding enhancer that acts as a transcription factor and regulates the expression of genes associated with the tumor cell cycle and homeostasis. It was previously reported that CEBPA may be a lipid-generating gene and that its expression increases synchronously with that of leptin patients with ovarian cancer, therefore impacting on their prognosis $(32,33)$.

In conclusion, the present study demonstrated that the transcription factor CEBPA activates leptin gene transcription by binding to its upstream promoter region. High levels of leptin may reduce the cytotoxic effect of PTX/TXT in ovarian cancer cells by activating EMT. Therefore, leptin has potential as a chemotherapeutic resistance predictor and/or as a novel therapeutic target in patients with epithelial ovarian cancer undergoing platinum plus PTX/TXT chemotherapy.

\section{Acknowledgments}

The authors would like to thank Dr Qin Yang, Dr Li-Peng Hu and Dr Miao Dai (Shanghai Cancer Institute, Renji Hospital, Shanghai Jiao Tong University School of Medicine) for their technical and material support.

\section{Funding}

The present study was supported by the National Natural Science Foundation of China (grant nos. 81472445 and 81672587 to RZ), the Scientific and Technological Innovation Act Program of Shanghai Science and Technology Commission (grant no. 14411973100 to RZ), the Scientific and Technological Innovation Act Program of Fengxian Science and Technology Commission (grant no. 20160908 to HL), and the National Natural Science Foundation of China (grant no. 81571401 to LQY).

\section{Availability of data and materials}

The datasets used and/or analyzed during the current study are available from the corresponding author on reasonable request.

\section{Authors' contributions}

FG performed all the experiments and was a major contributor in writing the manuscript. HZ analyzed the prediction. LY, SJ, $\mathrm{HL}, \mathrm{XX}$ and $\mathrm{CZ}$ participated in the design of the experiments and the revision of the manuscript. RZ contibuted the most to the study design, and PJ participated in the design and guidance of the project. All authors read and approved the final manuscript.

\section{Ethics approval and consent to participate}

Not applicable

\section{Patient consent for publication}

Not applicable.

\section{Competing interests}

The authors declare that they have no competing interests.

\section{References}

1. Torre LA, Bray F, Siegel RL, Ferlay J, Lortet-Tieulent J and Jemal A: Global cancer statistics, 2012. CA Cancer J Clin 65: 87-108, 2015

2. Leung CS, Yeung TL, Yip KP, Wong KK, Ho SY, Mangala LS, Sood AK, Lopez-Berestein G, Sheng J, Wong ST, et al: Cancer-associated fibroblasts regulate endothelial adhesion protein LPP to promote ovarian cancer chemoresistance. J Clin Invest 128: 589-606, 2018.

3. Siegel RL, Miller KD and Jemal A: Cancer statistics, 2016. CA Cancer J Clin 66: 7-30, 2016.

4. Dasari S and Tchounwou PB: Cisplatin in cancer therapy: Molecular mechanisms of action. Eur J Pharmacol 740: 364-378, 2014.

5. Batista ML Jr, Olivan M, Alcantara PS, Sandoval R, Peres SB, Neves RX, Silverio R, Maximiano LF, Otoch JP and Seelaender M: Adipose tissue-derived factors as potential biomarkers in cachectic cancer patients. Cytokine 61: 532-539, 2013.

6. Andò S, Barone I, Giordano C, Bonofiglio D and Catalano S: The multifaceted mechanism of leptin signaling within tumor microenvironment in driving breast cancer growth and progression. Front Oncol 4: 340, 2014.

7. Chang CC, Wu MJ, Yang JY, Camarillo IG and Chang CJ: Leptin-STAT3-G9a signaling promotes obesity-mediated breast cancer progression. Cancer Res 75: 2375-2386, 2015.

8. Visintin I, Feng Z, Longton G, Ward DC, Alvero AB, Lai Y, Tenthorey J, Leiser A, Flores-Saaib R, Yu H, et al: Diagnostic markers for early detection of ovarian cancer. Clin Cancer Res 14: 1065-1072, 2008

9. Serin IS, Tanriverdi F, Yilmaz MO, Ozcelik B and Unluhizarci K: Serum insulin-like growth factor (IGF)-I, IGF binding protein (IGFBP)-3, leptin concentrations and insulin resistance in benign and malignant epithelial ovarian tumors in postmenopausal women. Gynecol Endocrinol 24: 117-121, 2008.

10. La Cava A and Matarese G: The weight of leptin in immunity. Nat Rev Immunol 4: 371-379, 2004.

11. Zhou W, Guo S and Gonzalez-Perez RR: Leptin pro-angiogenic signature in breast cancer is linked to IL-1 signalling. Br J Cancer 104: 128-137, 2011.

12. Vernooy JHJ, Ubags ND, Brusselle GG, Tavernier J, Suratt BT, Joos GF, Wouters EF and Bracke KR: Leptin as regulator of pulmonary immune responses: Involvement in respiratory diseases. Pulm Pharmacol Ther 26: 464-472, 2013. 
13. Geng Y, Wang J, Wang R, Wang $\mathrm{K}$, Xu Y, Song G, Wu C and Yin Y: Leptin and HER-2 are associated with gastric cancer progression and prognosis of patients. Biomed Pharmacother 66: 419-424, 2012

14. Harbuzariu A, Rampoldi A, Daley-Brown DS, Candelaria P, Harmon TL, Lipsey CC, Beech DJ, Quarshie A, Ilies GO and Gonzalez-Perez RR: Leptin-Notch signaling axis is involved in pancreatic cancer progression. Oncotarget 8: 7740-7752, 2017.

15. Beales ILP, Garcia-Morales C, Ogunwobi OO and Mutungi G: Adiponectin inhibits leptin-induced oncogenic signalling in oesophageal cancer cells by activation of PTP1B. Mol Cell Endocrinol 382: 150-158, 2014.

16. Jin JH, Kim HJ, Kim CY, Kim YH,Ju W and Kim SC: Association of plasma adiponectin and leptin levels with the development and progression of ovarian cancer. Obstet Gynecol Sci 59: 279-285, 2016.

17. Housa D, Housová J, VernerováZ and Haluzík M: Adipocytokines and cancer. Physiol Res 55: 233-244, 2006.

18. Trisolini C, Albrizio M, Roscino MT, Pantaleo M, Rizzo A and Sciorsci RL: Leptin and queen ovary: New insights about ovulation. Res Vet Sci 94: 707-710, 2013.

19. Yu Y, Gaillard S, Phillip JM, Huang TC, Pinto SM, Tessarollo NG, Zhang Z, Pandey A, Wirtz D, Ayhan A, et al: Inhibition of spleen tyrosine kinase potentiates paclitaxel-induced cytotoxicity in ovarian cancer cells by stabilizing microtubules. Cancer Cell 28: 82-96, 2015.

20. Khan IU, Khan RU, Asif H, Alamgeer, Khalid SH, Asghar S, Saleem M, Shah KU, Shah SU, Rizvi SAA and Shahzad Y: Co-delivery strategies to overcome multidrug resistance in ovarian cancer. Int J Pharm 533: 111-124, 2017.

21. Monk BJ, Herzog TJ and Tewari KS: Evolution of chemosensitivity and resistance assays as predictors of clinical outcomes in epithelial ovarian cancer patients. Curr Pharm Des 22: 4717-4728, 2016

22. Fletcher JI, Haber M, Henderson MJ and Norris MD: ABC transporters in cancer: More than just drug efflux pumps. Nat Rev Cancer 10: 147-156, 2010.

23. Iyer AK, Singh A, Ganta S and Amiji MM: Role of integrated cancer nanomedicine in overcoming drug resistance. Adv Drug Deliver Rev 65: 1784-1802, 2013.

24. Jabr-Milane LS, van Vlerken LE, Yadav S and Amiji MM: Multi-functional nanocarriers to overcome tumor drug resistance. Cancer Treat Rev 34: 592-602, 2008.
25. Mahmood K, Zia KM, Zuber M, Salman M and Anjum MN: Recent developments in curcumin and curcumin based polymeric materials for biomedical applications: A review. Int J Biol Macromol 81: 877-890, 2015

26. Cuello MA, Kato S and Liberona F: The impact on high-grade serous ovarian cancer of obesity and lipid metabolism-related gene expression patterns: The underestimated driving force affecting prognosis. J Cell Mol Med 22: 1805-1815, 2018.

27. Otero M, Lago R, Lago F, Reino JJ and Gualillo O: Signalling pathway involved in nitric oxide synthase type II activation in chondrocytes: Synergistic effect of leptin with interleukin-1. Arthritis Res Ther 7: R581-R591, 2005.

28. Vuolteenaho K, Koskinen A, Kukkonen M, Nieminen R, Päivärinta U, Moilanen T and Moilanen E: Leptin enhances synthesis of proinflammatory mediators in human osteoarthritic cartilage-mediator role of NO in leptin-induced PGE2, IL-6, and IL-8 production. Mediators Inflamm 2009: 345838, 2009.

29. Liang SQ, Marti TM, Dorn P, Froment L, Hall SR, Berezowska S, Kocher G, Schmid RA and Peng RW: Blocking the epithelial-to-mesenchymal transition pathway abrogates resistance to anti-folate chemotherapy in lung cancer. Cell Death Dis 6: e1824, 2015.

30. Mitra A, Mishra L and Li S: EMT, CTCs and CSCs in tumor relapse and drug-resistance. Oncotarget 6: 10697-10711, 2015.

31. Sánchez-Tilló E, Fanlo L, Siles L, Montes-Moreno S, Moros A, Chiva-Blanch G, Estruch R, Martinez A, Colomer D, Győrffy B, et al: The EMT activator ZEB1 promotes tumor growth and determines differential response to chemotherapy in mantle cell lymphoma. Cell Death Differ 21: 247-257, 2014.

32. Kim E, Lim S, Kim M, Yoo S and Kim Y: Phyllodulcin, a natural sweetener, regulates obesity-related metabolic changes and fat browning-related genes of subcutaneous white adipose tissue in high-fat diet-induced obese mice. Nutrients 9: pii: E1049, 2017.

33. Konopka B, Szafron LM, Kwiatkowska E, Podgorska A, Zolocinska A, Pienkowska-Grela B, Dansonka-Mieszkowska A, Balcerak A, Lukasik M, Stachurska A, et al: The significance of c. $690 \mathrm{G}>\mathrm{T}$ polymorphism (rs34529039) and expression of the CEBPA gene in ovarian cancer outcome. Oncotarget 7: 67412-67424, 2016.

This work is licensed under a Creative Commons Attribution-NonCommercial-NoDerivatives 4.0 International (CC BY-NC-ND 4.0) License. 